\title{
Finite Element Prediction of Fatigue Damage Growth in Cancellous Bone
}

Ridha Hambli, Sana Frikha

PRISME laboratory, EA4229, University of Orleans

Polytech’ Orléans, 8, Rue Léonard de Vinci 45072 Orléans, France

Hechmi Toumi

University of Orleans, I3MTO Laboratory, EA 4708, Hospital of Orleans, 1 rue Porte Madeleine, F45032, Orléans, France

João Manuel R. S. Tavares

Instituto de Ciência e Inovação em Engenharia Mecânica e Engenharia Industrial, Departamento de

Engenharia Mecânica, Faculdade de Engenharia, Universidade do Porto, Rua Dr. Roberto Frias s/n, Porto, Portugal

tavares@fe.up.pt

Phone: +351225081487

Fax: +35122 5081445

(Corresponding author) 


\title{
Finite Element Prediction of Fatigue Damage Growth in Cancellous Bone
}

\begin{abstract}
Cyclic stresses applied to bones generate fatigue damage that affects the bone stiffness and its elastic modulus. This paper proposes a finite element model for the prediction of fatigue damage accumulation and failure in cancellous bone at continuum scale. The model is based on continuum damage mechanics and incorporates crack closure effects in compression. The propagation of the cracks is completely simulated throughout the damaged area. In this case, the stiffness of the broken element is reduced by $98 \%$ to ensure no stress carrying capacities of completely damaged elements. Once a crack is initiated, the propagation direction is simulated by the propagation of the broken elements of the mesh. The proposed model suggests that damage evolves over a real physical time variable (cycles). In order to reduce the computation time, the integration of the damage growth rate is based on the cycle blocks approach. In this approach, the real number of cycles is reduced (divided) into equivalent blocks of cycles. Damage accumulation is computed over the cycle blocks and then extrapolated over the corresponding real cycles. The results show a clear difference between local tensile and compressive stresses on damage accumulation. Incorporating stiffness reduction also produces a redistribution of the peak stresses in the damaged region, which results in a delay in damage fracture.
\end{abstract}

Keywords: Cancellous bone, Microdamage, Fatigue, Microcracks, Finite element 


\section{Introduction}

Bone resorption has been suggested as the primary failure mode of femoral prostheses. All prostheses are supported by cancellous bone, and it is well admitted that medium-term migration of implants may be due to the failure of cancellous bone caused by cyclic stress (McNamara et al., 1997, Taylor and Tanner, 1997). Cyclic loading accelerates failure in cancellous bone, leading to bone resorption. As a result, the rate of migration will vary with the ability of vascularised cancellous bone to repair damage (McNamara et al., 1997, Taylor and Tanner, 1997). If damage exceeds repair, then it is likely that migration will continue, although at a slower rate than during the initial phase. Also, it will cease if repair equals or exceeds the rate of tissue damage.

The adaptation of bone to cyclic fatigue loads involves a complex physiological response that is targeted to local sites of microdamage. The mechanisms that regulate this process are complex and not well understood. However, it has been suggested that osteocytes produce a signal proportional to mechanical loading by sensing strain and fatigue microcracks and transmit signals activating remodelling to remove bone where the damage is excessive.

Accurate assessment of microdamage is important to evaluate bone remodelling (Burr, 1993; Burr and Hooser, 1995; McNamara and Prendergast, 2007b; Prendergast and Taylor, 1994; Hambli et al., 2009; Hambli, 2011a, b), osteoporotic therapeutics (Hirano et al., 1997) and implant migration and loosening (McNamara et al., 1997; Taylor and Tanner, 1997).

When the loading cycles have been determined, the fatigue analysis of bone can be performed. The methods for fatigue analysis are most frequently based on the relation between deformations, stresses and number of loading cycles, and are usually modified to fit the nature of the stress cycle (Sobelman et al., 2004; Zioupos et al., 1996). The number of stress cycles required for a fatigue crack to appear can be determined iteratively with the strain-life method $\varepsilon-N$.

For purely elastic behaviour, various uncoupled models have been developed to describe the fatigue life. In general, the more complex combined shear and normal strain/stress parameters using critical plane analysis for non-proportional loading are the most effective (Burr, 1993; Burr and Hooser, 1995; McNamara and Prendergast, 2007a; Prendergast and Taylor, 1994). 
In their work, Sobelman et al. (2004) fatigued 20 beams of femoral cortical bone from elderly men and women in load-controlled four-point bending with a strain range of 3000 or 5000 microstrains. They proposed an experimental Manson-Coffin type relationship between the specific deformation and the number of loading cycles.

In order to simulate the initiation and propagation of microcrack fatigue in cancellous bone, a new finite element model has been developed here. The proposed model is based on continuum damage mechanics that describes in a fully coupled way the damaging process as a result of cyclic strains applied to bone up to the appearance of microcracks.

Propagation of the cracks is completely simulated throughout the damaged area. In this case, the stiffness of the broken elements is reduced by $98 \%$ to ensure no stress carrying capacities of completely damaged elements. Once a crack has been initiated, the propagation direction is simulated by the propagation of the broken elements of the mesh. The proposed model suggests that damage evolves over a real physical time variable (cycles). In order to reduce the computational time required for the cyclic loading steps of the simulation, the cycle blocks approach was implemented. It consists in dividing the real number of cycles into equivalent cycle blocks. Damage accumulation is computed over the cycle blocks and then extrapolated over the corresponding real cycles.

\section{Fatigue damage modelling}

Experimental investigations have linked bone adaptation to microdamage. It was suggested by Taylor and Tanner (1997) that microcrack detection is related to rupturing of the cellular material itself due to crack face displacements. Using specific cell staining techniques, it was confirmed that relative crack displacements are capable of tearing cell processes between neighbouring osteocytes. There are two possible ways in which cells may be fractured. Firstly, cells immediately ahead of the crack tip may experience an excessive strain which breaks them; secondly, the relative displacements of the crack faces may be sufficient to fracture cellular processes. Ruptured cell processes may directly secrete passive and active components in the extracellular matrix, triggering a repair response (Taylor and Tanner, 1997). 
Continuum Damage Mechanics (CDM) can be used to follow the bone damage process as results of cyclic strains applied to bone up to the appearance of microcracks. Lemaitre (1985) and Chaboche (1981) suggested that damage in CDM is a state variable that takes into account the degradation of material which results in stiffness reduction. The damage variable can also be related to the physical modifications in the material such as initiation and propagation of microcracks.

Elasticity based damage models of high-cycle fatigue assume a decrease in elastic stiffness as the damage grows. This loss of stiffness can indeed be measured in bone and other materials.

Taylor et al. (2002) presented a preliminary study to simulate the fatigue behaviour of cancellous bone based on the assumption that the fatigue behaviour of trabecular bone is similar to that of cortical bone using a combined CDM and Finite Element Modelling. They examined the influence of material property degradation on the overall fatigue behaviour of a trabecular bone segment. Briefly, their work suggested that for all but the simplest load cases, the accumulation of permanent strain plays an important role in reducing localised high stresses and redistributing the load to the surrounding tissue.

Fatigue damage phenomena are localized at the trabeculae micro-scale and damage effects lead to the formation of microcracks which propagate during loading cycles till complete fracture of the trabeculae as shown in Figure 1.
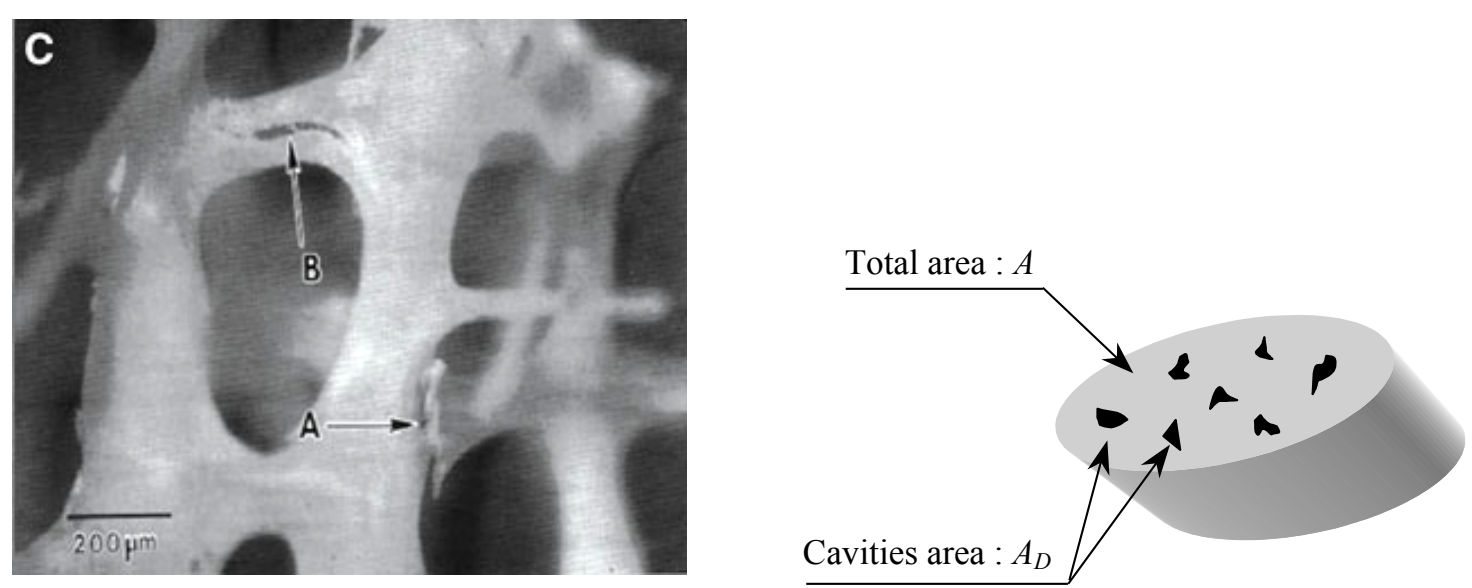

Figure 1. Microcracks in trabeculae (adapted from Fyhrie and Schaffler, 1994). 
In CDM (Figure 1), the isotropic damage variable $(D)$ is defined by the ratio between the total area of the microcracks and cavities $\left(A_{D}\right)$ and the overall sectional area of an elementary material-volume $(A)$ : $D=\frac{A_{D}}{A}$

\section{Damage evolution law}

When dealing with loading histories composed of well-defined discrete cycles, an evolution law in terms of the number of cycles and their amplitudes is often considered more practical in the literature. The number of cycles $(N)$ is then regarded as a continuous time variable and the growth of damage, which occurs during discrete time intervals within a cycle, is spread to a continuous evolution over the entire cycle. Such a cycle based formulation can be obtained in the form of:

$$
\frac{\partial D}{\partial N}=f\left(\frac{N}{N_{f}}\right)
$$

where $N_{f}$ denotes the cycle at failure.

For high cycle fatigue under purely elastic strain without coupling between heat dissipation and mechanical dissipation, it is possible to write (Lemaitre, 1985):

$$
\partial D=\left[1-(1-D)^{\beta+1}\right]^{\alpha}\left(\frac{\sigma_{e q M}-\bar{\sigma}}{M(1-D)}\right)^{\beta} \partial N
$$

where $\alpha$ is a parameter depending mainly on the loading conditions, $\beta$ and $M$ are material parameters that can be obtained from experimental fatigue tests from S-N curves, and $\sigma_{e q M}$ and $\bar{\sigma}$ are the equivalent maximum peak stress and the mean stress, respectively.

The integration of Eq. (3) leads to Chaboche's model expressed as (Chaboche, 1981):

$$
D=1-\left[1-\left(\frac{N}{N_{f}}\right)^{\frac{1}{1-\alpha}}\right]^{\frac{1}{1+\beta}} \text {. }
$$


Fatigue accumulation of bone depends on the loading conditions. Tension or compression loads generate two different damage evolutions due to static rupture of bone and crack closure of microcracks in compression. To take into account such effects, one way is to consider two different values for $N_{f}$ that can be obtained from experimental laws. In this work, the following relations based on experimental investigations conducted by Martin et al. (1998) were implemented:

$N_{f}^{c}=1.479 \times 10^{-21} \mathcal{E}^{-10.3}$, for compressive loads,

$N_{f}^{t}=3.630 \times 10^{-32} \varepsilon^{-14.1}$, for tensile loads,

where $\varepsilon$ is the amplitude of applied microstrain.

The parameter $\alpha$ is calculated as:

$\alpha=1-\eta\left\langle\frac{\sigma_{e q M}-\sigma_{D}}{\sigma_{u}-\sigma_{e q M}}\right\rangle$,

where $\eta$ is a material parameter obtained from S-N experiments, $\sigma_{e q M}, \sigma_{D}$ and $\sigma_{u}$ are the equivalent maximum peak stress, the fatigue limit and the ultimate stress, respectively (Figure 2), and the operator $\langle\ldots\rangle$ denotes: $\langle x\rangle=x$ if $x \geq 0$ and $\langle x\rangle=0$ if $x<0$.

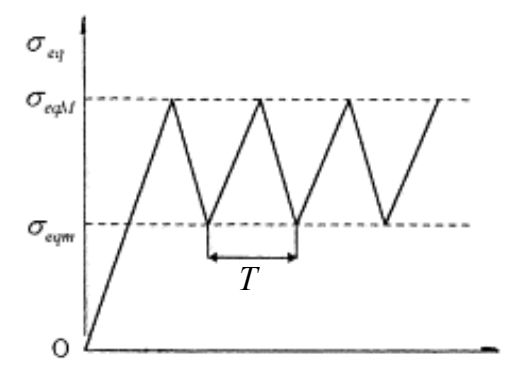

Figure 2. Alternation of $\sigma_{e q}$ during cycle loading.

A major drawback of cumulative damage accumulation models is the computational cost associated with modelling every loading cycle. In order to reduce the computation time, the integration of the damage growth rate is based on the cycle blocks approach. In this approach, the real number of cycles is reduced (divided) into equivalent cycle blocks (Figure 3). Damage accumulation is computed over the cycle blocks and extrapolated over the corresponding real cycles. 


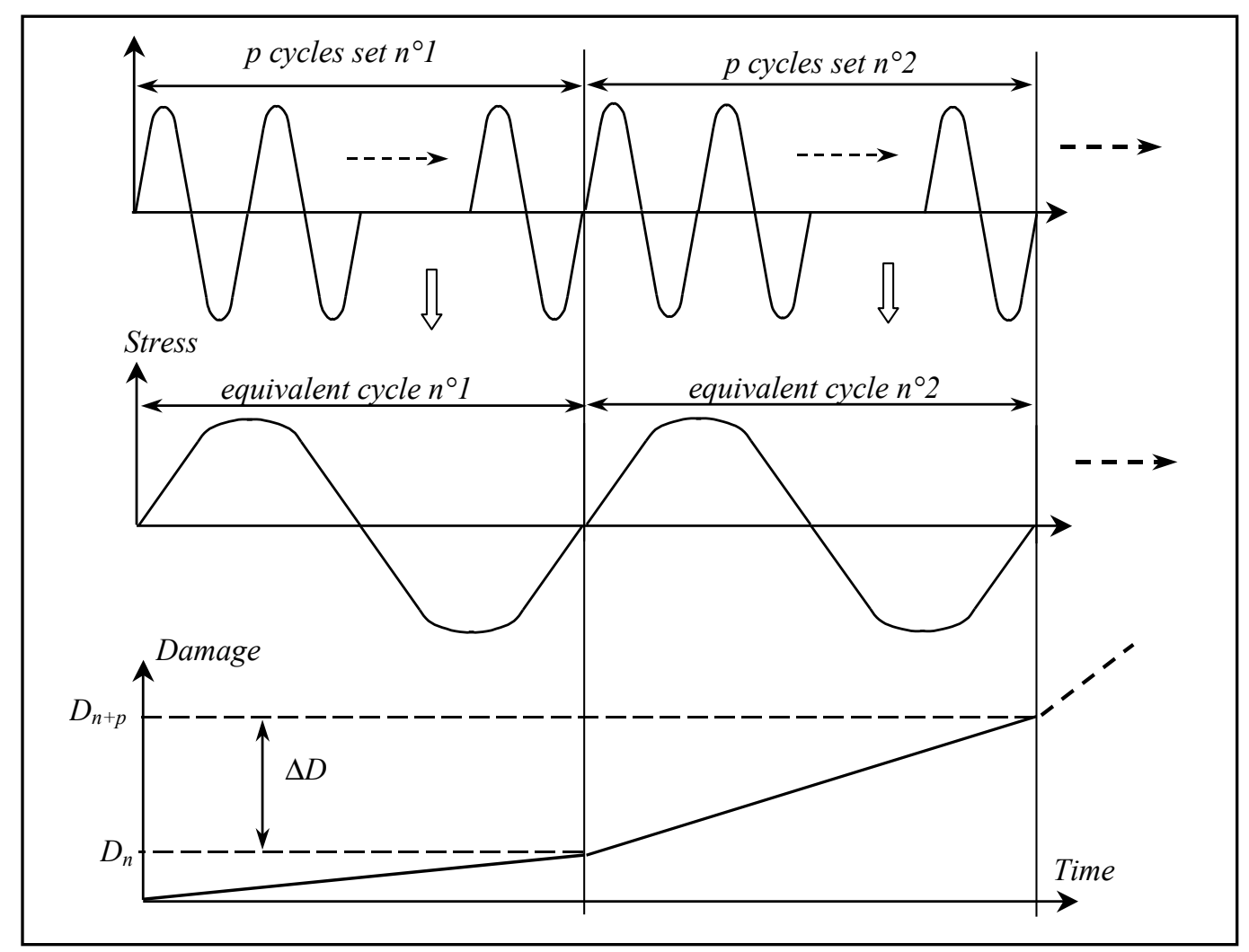

Figure 3. Cycle block sets approach for damage accumulation computation.

Within the framework of the cycle blocks approach, damage evolution can be obtained by:

$D_{n+p}=D_{n}+\Delta D$,

where $D_{n+p}$ is the damage at iteration $n+p$, with $p$ denoting the number of cycles in one block set, $D_{n}$ is the damage at iteration $n$, and $\Delta D$ is the damage increment computed for one jump of $p$ cycles.

Further, to compute the damage at every cycle, we extrapolate the damage state using:

$D_{n+1}=D_{n}+\frac{\Delta D}{p}$. 


\section{Finite element model}

In order to predict fatigue damage effects, a new finite element model has been developed and implemented in ABAQUS (Dassault Systèmes, USA) using the UMAT routine. Figure 4 shows the flowchart of the coupled analysis.

If damage at an integration point reaches a critical value $D_{R}$, the modulus is reduced by $98 \%$. As a result, the resorption was simulated by setting the bone density at $0.08 \mathrm{~g} / \mathrm{cm}^{3}$ (Hambli and Thurner, 2013; Hambli, 2013).

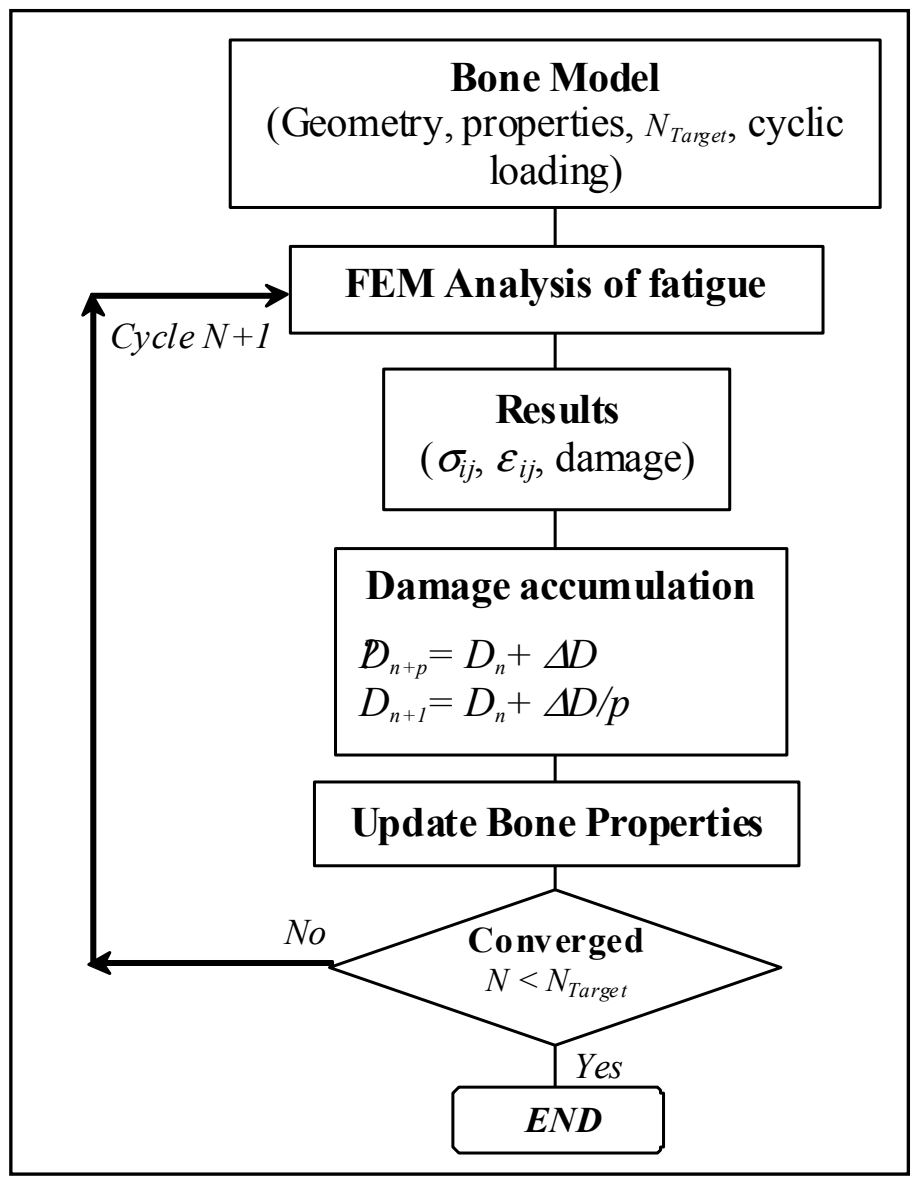

Figure 4. Flowchart of coupled algorithm for predicting bone damage effects.

To simulate the influence of fatigue, the finite element model is run in alternating load and unload increments. At each load increment, damage and bone material properties are updated at each integration point in every element. The simulation of every loading cycle of a long life fatigue problem would have been computationally expensive; therefore, a procedure was adopted to approximate many cycles with a single equivalent cycle. 
The bone material parameters used in the present work are indicated in Table 1.

Table 1. Bone parameter values used for fatigue finite element simulation.

\begin{tabular}{|lcc|}
\hline \multicolumn{1}{|c|}{ Parameter } & Notation & Value \\
\hline Initial Elastic modulus & $E_{0}(\mathrm{MPa})$ & 15000 \\
\hline Poisson ratio & $V$ & 0.3 \\
\hline Initial density & $\rho\left(\mathrm{g} / \mathrm{cm}^{3}\right)$ & 1.0 \\
\hline Fatigue parameter & $\eta$ & 0.7 \\
\hline Fatigue exponent & $\beta$ & 0.4 \\
\hline Ultimate stress & $\sigma_{u}(\mathrm{MPa})$ & 133 \\
\hline Fatigue limit & $\sigma_{D}(\mathrm{MPa})$ & 60 \\
\hline
\end{tabular}

\section{Discussion}

To qualitatively validate the proposed bone fatigue model, a local 2D simulation was performed corresponding to a micro trabecular bone shape as shown in Figure 5.

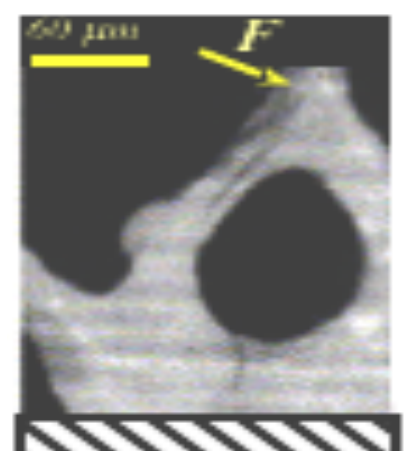

Figure 5. Bone fatigue microcrack model.

A mesh is generated using 5700 four-node second order plane-stress elements with an average mesh size of about 15 microns.

In the constitutive law of conventional continuum damage mechanics, there is no intrinsic material characteristic length, which means that crack propagation results are dependent on the mesh size (Tsouknidas et al., 2015). Several approaches were developed in the past to identify the optimum mesh density that would ensure accuracy of the results in terms of model verification (Viceconti et al., 2005). This is usually achieved with a mesh independent pattern, ensuring that coarsening of the mesh does not disturb the stress field by more than $2 \%$ (Tsouknidas et al., 2015). In the current work, when the damage parameter reaches a critical value inside an element, the element mechanical 
contribution to the stiffness matrix is reduced by a factor of $98 \%$, leading to redistribution of the stress state in the crack tip. Once a crack has been initiated, the propagation direction is simulated by the propagation of the broken elements of the mesh. The average crack length found in bones is typically 100 microns (Burr, 1993; Taylor and Lee, 2002). In the present model, this size corresponds to the mesh characteristic length at trabeculae level of a set of about 7 finite elements (15 microns/element). Hence, to prevent mesh dependence that generally affects the damage propagation rate, numerical fatigue fracture occurs when the damage value reaches a critical value at a set of 7 serial broken elements (a crack length of about 100 microns). The proposed mesh-dependency regularization method was successfully applied in previous work dealing with crack propagation, and ensures non-dependency of the fracture results on the finite element mesh (Hambli, 2011a, b).

A cyclic force with an amplitude of $50 \mathrm{~N}$ was applied to generate fatigue damage during 200 iterations (days); under these conditions, the local deformation exceeds $3500 \mu \varepsilon$ in some elements. Figure 6 depicts the contour of fatigue damage obtained for different iterations.

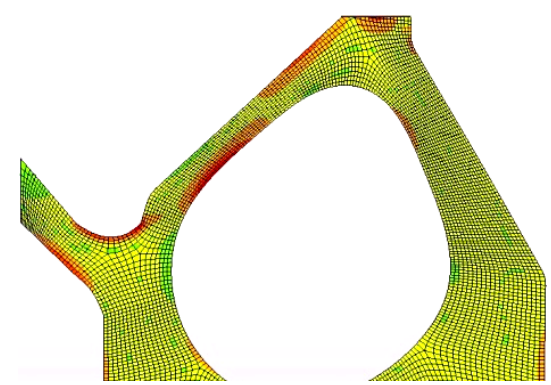

(a) Cycles $=1 . \mathrm{E}+3$

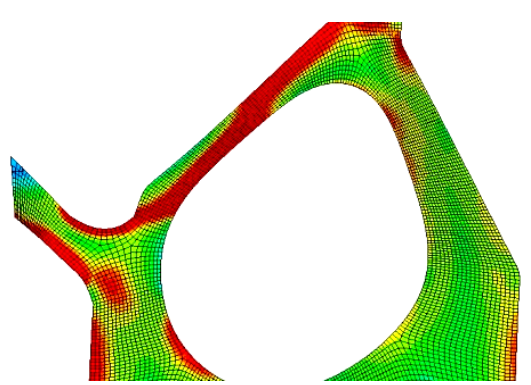

(c) Cycles $=1 . \mathrm{E}+6$

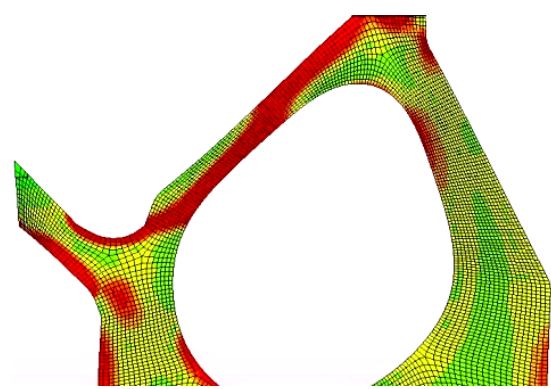

(b) Cycles $=1 . \mathrm{E}+4$

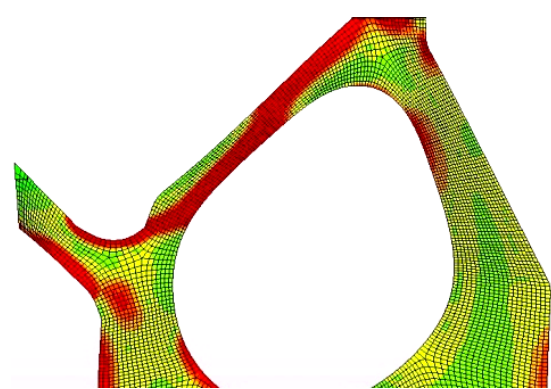

(d) Cycles $=1 . \mathrm{E} 8$

Figure 6. Simulated contour of fatigue damage at different cycles.

Figure 7 shows the crack initiation and propagation obtained by the simulation performed. 


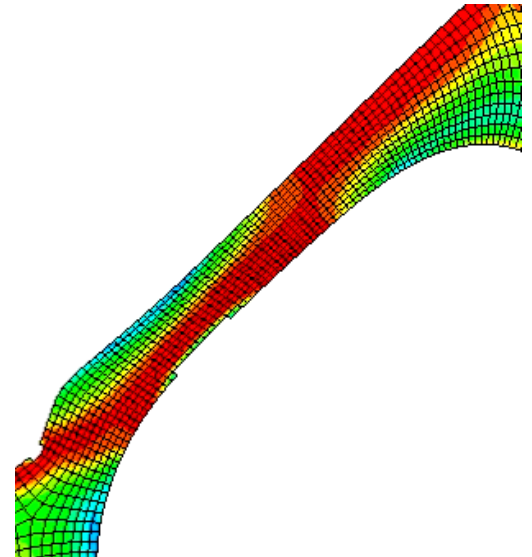

(a) Cycles $=1 . E+6$

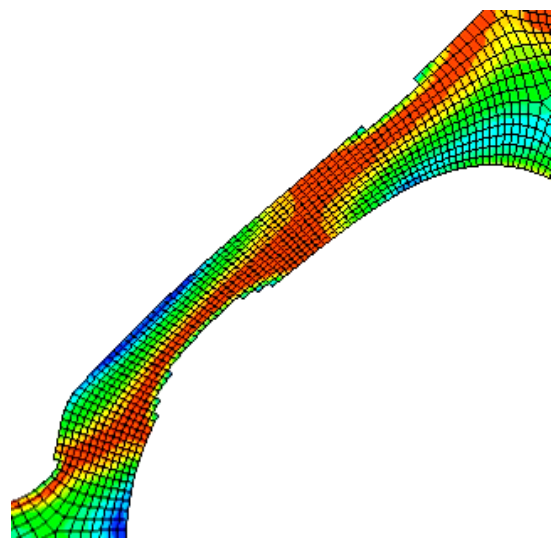

(b) Cycles $=1 . \mathrm{E}+7$

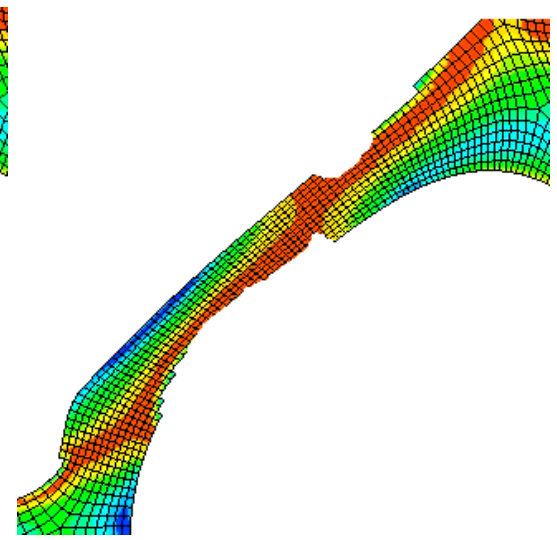

(c) Cycles $=1 . \mathrm{E}+8$

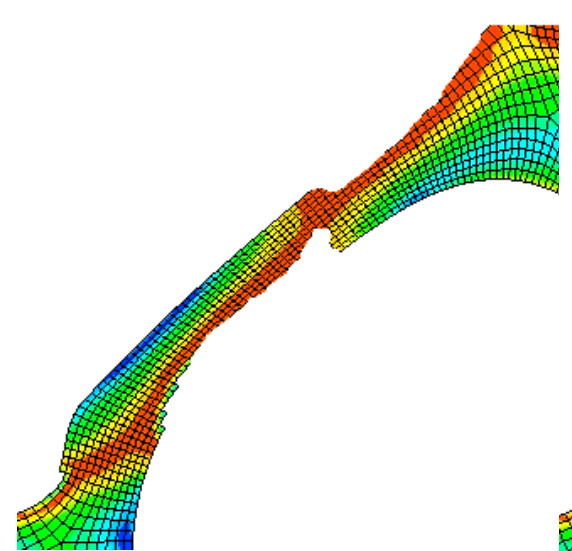

(d) Cycles $=1.5 \mathrm{E}+8$

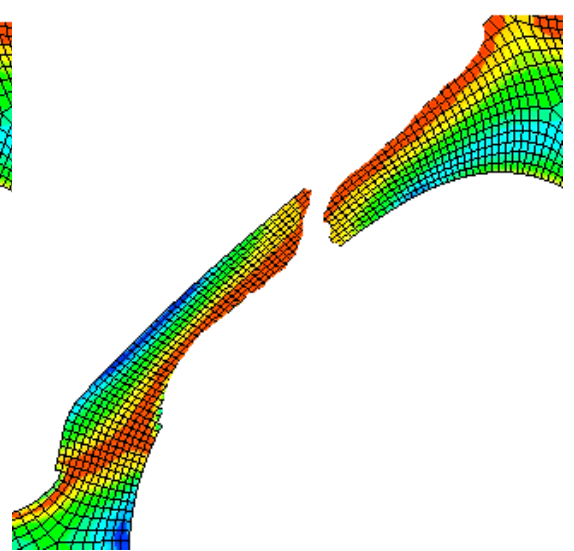

(e) Cycles $=1.7 \mathrm{E}+8$

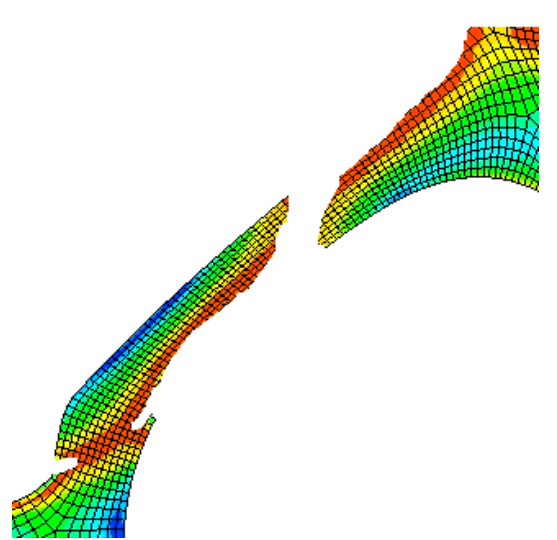

(f) Cycles $=1.8 \mathrm{E}+8$

Figure 7. Simulated crack propagation at different cycles.

The proposed model was able to predict the sites of bone fatigue microcrack growth located in the maximally damaged zones. The predicted fatigue damage of single trabeculae leads to crack initiation and propagation, which appears to be local, in agreement with experimental observations (Dendorfer et al., 2009).

Figure $8 \mathrm{~b}$ depicts the variation of damage versus $x$ (Fig. 8a) distance resulting from the simulation conducted.

The results reveal that the maximum of damage is reached in tensile zones. This is due to the weakness of bone in tension. This damage variation causes a drop in stresses as can be observed in Figure 9. In distance corresponding to the highest damage value $(x=75 \%)$, the stress drop is maximum. 

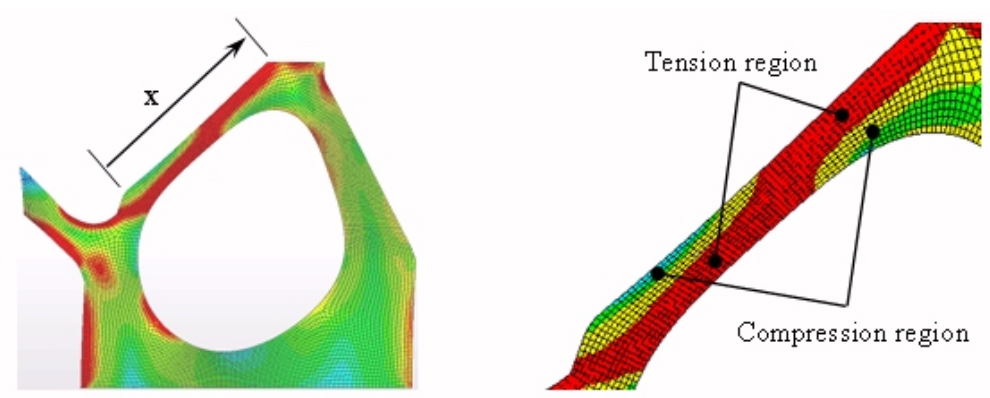

(a) Localized damage in tensile regions of the trabeculae (cycle $=1 . \mathrm{E}+05)$.

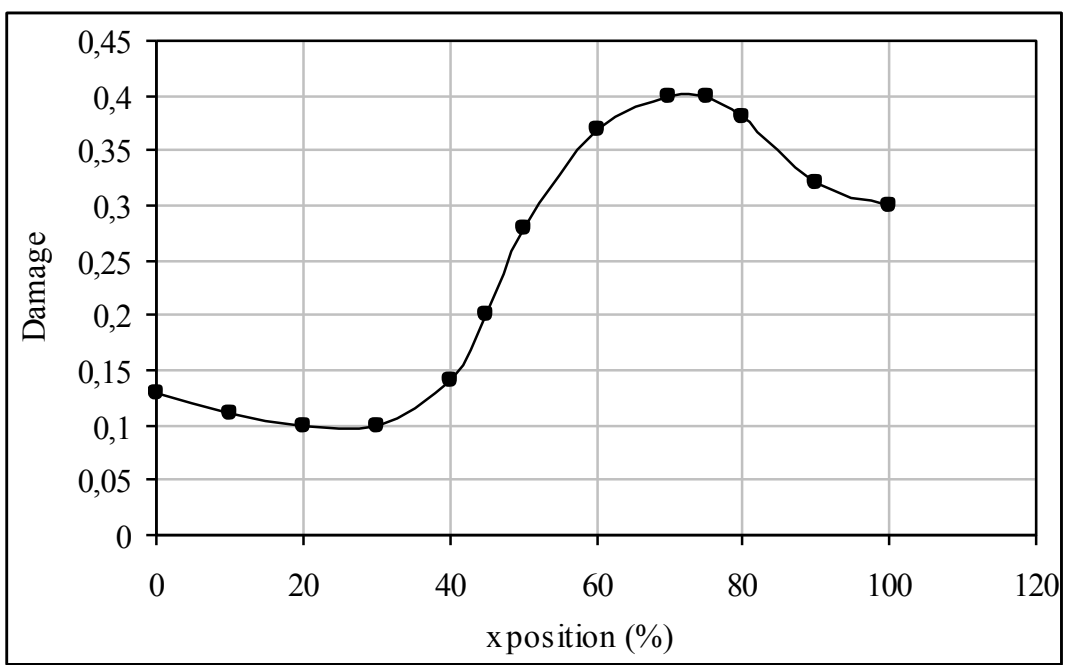

(b) Damage variation.

Figure 8. Damage distribution in one trabecula versus $x$ distance $($ cycle $=1 . \mathrm{E}+05)$.

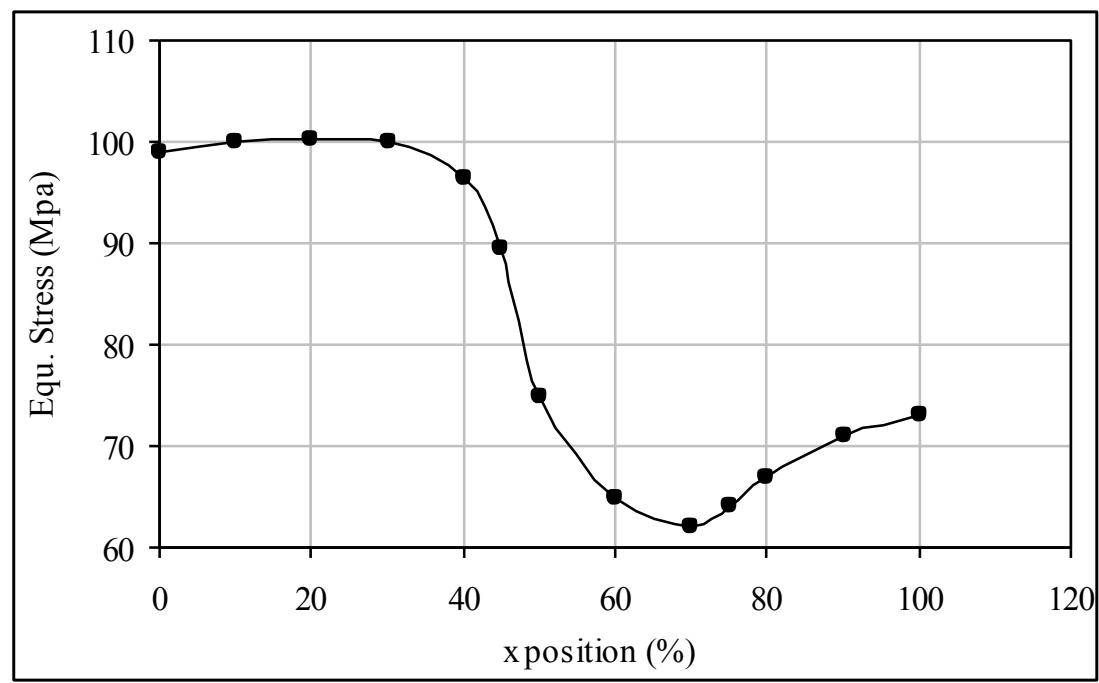

Figure 9. von Mises equivalent stress distribution in one trabecula versus $x$ distance (cycle $=1 . \mathrm{E}+07)$.

It has been suggested that fatigue damage, in the form of microcracks, may act as a signal to regulate bone remodeling. These microcracks, which result in the disruption of osteocytic communication via the bone canalicular network (Hambli and Rieger, 2011; Hambli et al., 2015) 
may, in fact, be an important stimulus, providing spatial regulation of the bone damage repair process (Burr, 1993; Taylor et al., 2007). The balance between local remodeling and the accumulation of trabecular bone fatigue microcracks is believed to play an important role in the maintenance of skeletal integrity. This occurs by the resorption of damaged bone by osteoclasts followed by new bone formation by osteoblasts.

In general, bone fatigue damage modeling is investigated from two perspectives: (i) Mechanical/Material approaches that use the FE method to model and simulate fatigue damage accumulation and growth in the bone under applied cyclic loading leading to crack formation, and (ii) Bone remodeling approaches aimed at simulating the fatigue damage repair.

Taylor et al. (2007) discussed some issues related to the two approaches. The main concern is that many experimental studies performed in recent years show that damage can be linked to several factors such as reduced stiffness, ageing, osteoporosis and its treatment. Nevertheless, comprehensive theoretical fatigue models that take all these factors into consideration are still lacking. It has also been reported that mechanical properties. The histological evidence indicates that fatigue damage occurs at the microstructural level (microcracks) and at ultrastructural levels (Barkaoui and Hambli, 2014; Barkaoui et al., 2015).

Most theoretical and numerical studies of bone damage evolution at the macroscopic level have focused on the development of continuum approaches in which the total damage is a scalar quantity defined either as the normalized number of cycles or in terms of the changes in elastic modulus and residual strain during life (Burr, 1993; Taylor et al., 2002; Hernandez et al., 2006; McNamara and Prendergast, 2007; Kosmopoulos et al., 2008, Hambli et al., 2009, 2011a). Fracture mechanics laws have also been applied to investigate crack growth in bone (Taylor and Lee, 2003). The limitation of fracture mechanics based approaches is that it is very difficult to predict the behavior of every crack explicitly in a large population of cracks.

In spite of the large number of FE studies dealing with bone damage fatigue accumulation, there is still a lack of practical and simple FE models that simulate the complete and realistic behavior of bone fatigue considering both damaging processes leading to complete fracture. The 
present model based on continuum damage mechanics advances previous ones by incorporating both fatigue damage accumulation and the fracturing process. In the proposed model, there is no difference between crack initiation and propagation. Both result from the failure of an element with a characteristic dimension (typical crack size). Thus, fatigue crack initiation and propagation are studied in a fully coupled way as a unified approach; the complete propagation of the fatigue cracks is simulated by the propagation of the completely damaged elements of the mesh. In addition, the proposed numerical model is built such a way that the damage evolves over a real physical time variable (cycles) based on the cycle blocks approach.

The results of the present study are in agreement with previous ones (Zioupos et al., 1996; Taylor et al., 2002). These studies examined cycles to failure vs. stress and damage vs. stress and showed that bone experiences mainly a loss in material stiffness and loss in strength.

Micro-CT FE models applied to trabecular bone specimens (Homminga et al., 2004; Hernandez et al., 2006, Dendorfer et al., 2009) showed that microdamage initiation occurs prior to apparent yield at relatively low local principal strains in compression. The authors suggested that local tissue yielding can, in fact, initiate at very low apparent strains and that the apparent mechanical properties are degenerated through these localized effects. Morgan et al. (2005) suggested that relatively small amounts of microdamage have a major effect on the mechanical properties of bone. The results in Fig. 7 show that fatigue microcracks accumulate in regions of strain concentration within the trabeculae.

This study showed that the current model is an enhanced, practical and simple one that can be applied to assess the effect of a given cyclic load on fatigue damage accumulation within bone. From a clinical point of view, the model may be applied as a diagnostic tool that can simulate the fatigue damage accumulation effects for individual patients in an early stage and investigate the consequences in terms of bone strength. Before potential clinical implementation, experimental validation by performing several experiments with different bone samples and boundary conditions is needed. 


\section{Conclusion}

A new finite element model has been proposed to predict fatigue damage and failure in cancellous bone at continuum scale. The model is based on continuum damage mechanics that describes the evolution of the damage process as a result of cyclic strains applied to bone up to the appearance of microcracks.

The proposed model suggests that damage evolves over a real physical time variable (cycles). In order to reduce the computation time, the integration of the damage growth rate is based on the cycle blocks approach. The idea is that the real number of cycles is reduced (divided) into equivalent cycle blocks. Damage accumulation is computed over the cycle blocks and extrapolated over the corresponding real cycles.

The results show a clear difference between local tensile and compressive stresses on damage accumulation. Incorporating stiffness reduction produces a redistribution of the peak stresses in the damaged region, which results in a delay of damage fracture.

The proposed model can be applied to the development of simulation of the trabecular bone remodeling process incorporating strain and damage coupled effects and the assessment of implant migration and loosening generated by damage.

\section{References}

Barkaoui A. and Hambli R., 2014, Nanomechanical properties of mineralized collagen microfibrils based on finite element method: biomechanical role of cross-links, Comp. Met. in Biomech. and Biomed. Eng., 17(14):1590-601.

Barkaoui A., Hambli R. and Manuel R.S. Tavares J., 2015, Effect of material and structural factors on fracture behaviour of mineralised collagen microfibril using finite element simulation, Comp. Met. in Biomech. and Biomed. Eng., 18(11):1181-1190.

Burr D.B., 1993, Remodeling and the repair of fatigue damage, Calcified Tissue Int, 53, Suppl 1:S7580; discussion S80-71. Epub 1993/01/01.

Burr D.B., Hooser M., 1995, Alterations to the En-Bloc Basic Fuchsin Staining Protocol for the Demonstration of Microdamage Produced in-Vivo, Bone, 17:431-433.

Chaboche J.L., 1981, Continuous Damage Mechanics - a Tool to Describe Phenomena before Crack Initiation. Nucl Eng Des., 64:233-247. 
Dendorfer S., Maier H.J. and Hammer J., 2009, Fatigue damage in cancellous bone: An experimenytal approach from continuum to micro scale, J. of mech. Behaviour of Biomed. Materials, 2:113-119.

Fyhrie DP., Schaffler MB., 1994, Failure Mechanisms in Human Vertebral Cancellous Bone, Bone, 15:105-109.

Hambli R., Soulat D., Gasser A. and Benhamou C.L. 2009. Strain-damage coupled algorithm for cancellous bone mechano-regulation with spatial function influence, Computer Methods in Applied Mechanics and Engineering, Vol. 198, Issues 33-36, 1, 2673-2682.

Hambli R., and Rieger R., 2011, Physiologically based mathematical model of transduction of mechanobiological signals by osteocytes. Biomech Model Mechanobiol., 11:1-2, 83-93

Hambli R., 2011a, Apparent Damage Accumulation in Cancellous Bone Using Neural Networks, Journal of the Mechanical Behavior of Biomedical Materials, 4:6, 868-878.

Hambli R., 2011b, Multiscale prediction of crack density and crack length accumulation in trabecular bone based on neural networks and finite element simulation, International Journal for Numerical Methods in Biomedical Engineering, (27):4, 461-475.

Hambli R., 2013. Micro-CT finite element model and experimental validation of trabecular bone damage and fracture, Bone, 56(2):363-74.

Hambli R. and Thurner P., 2013, Finite element prediction with experimental validation of damage distribution in single trabeculae during three-point bending tests, J Mech Behav Biomed Mater., 27:94-106.

Hambli R., Almitani K.H., Chamekh A., Toumi H., Tavares J.M., 2015, A theory for bone resorption based on the local rupture of osteocytes cells connections: A finite element study, Math Biosci., 30(262C):46-55.

Hernandez C.J., Gupta A., Keaveny T.M., 2006. A biomechanical analysis of the effects of resorption cavities on cancellous bone strength, J. Bone. Miner. Res., 21(8):1248-1255.

Hirano T., Turner C.H., Burr D.B., 1997. Effect of etidronate (EHDP) on bone turnover, microdamage accumulation and bone strength in dogs, J. of Bone and Mineral Research, 12:S484-S484.

Homminga J., Van Rietbergen B., Lochmueller E. M., Weinans H., Eckstein F., Huiskes R., 2004. The osteoporotic vertebral structure is well adapted to the loads of daily life, but not to infrequent “error" loads, Bone, 34(3):510-516.

Kosmopoulos V., Schizas C., Keller T.S., 2008. Modeling the onset and propagation of trabecular bone microdamage during low-cycle fatigue, Journal of Biomechanics, 41:515-522.

Lemaitre J., 1985. A Continuous Damage Mechanics Model for Ductile Fracture, J Eng Mater-T Asme., 107:83-89.

Martin R.B., Burr D.R., Sharkey N.A., 1998, Skeletal Tissue Mechanics, New York: Springer. 
McNamara B.P., Taylor D, Prendergast P.J., 1997, Computer prediction of adaptive bone remodelling around noncemented femoral prostheses: the relationship between damage-based and strain-based algorithms, Med Eng Phys., 19:454-463.

McNamara L.M., Prendergast J.P., 2007, Bone remodeling algorithms incorporating both stain and microdamage stimuli, Journal of Biomechanics, 60:1381-1391.

Morgan E.F., Yeh O.C. and Keaveny T.M., 2005, Damage in trabecular bone at small strains, Eur. J. Morphol., 42(1-2):13-21.

Prendergast P.J., Taylor D., 1994, Prediction of Bone Adaptation Using Damage Accumulation, Journal of Biomechanics, 27:1067-1076.

Sobelman O.S., Gibeling J.C., Stover S.M., Hazelwood S.J., Yeh O.C., Shelton D.R., Martin R.B., 2004, Do microcracks decrease or increase fatigue resistance in cortical bone?, Journal of Biomechanics, 37:1295-1303.

Taylor M., Tanner K.E., 1997, Fatigue failure of cancellous bone: A possible cause of implant migration and loosening, J Bone Joint Surg Br., 79B:181-182.

Taylor M., Cotton J., Zioupos P., 2002, Finite element simulation of the fatigue behaviour of cancellous bone, Meccanica, 37:419-429.

Taylor D. and Lee T.C., 2003, A crack growth model for the simulation of fatigue in bone, International Journal of Fatigue, 2:387-395.

Taylor D., Hazenberg J.G. and Clive Lee T., 2007, Living with cracks: Damage and repair in human bone, Nature Materials, 6:263-268.

Tsouknidas A., Maliaris G., Savvakis S., Michailidis N., 2015, Anisotropic post-yield response of cancellous bone simulated by stress-strain curves of bulk equivalent structures, Comput Methods Biomech Biomed Engin., 18(8):839-46.

Viceconti M., Olsen S., Nolte LP., Burton K., 2005, Extracting clinically relevant data from finite element simulations, Clin Biomech, 20(5):451-4.

Zioupos P., Wang X.T., Currey J.D., 1996, Experimental and theoretical quantification of the development of damage in fatigue tests of bone and antler, Journal of Biomechanics, 29(8):9891002. 